\title{
Lope de Vega, el tabaco y los cuernos: dos notas sobre polémica y burla en las Rimas de Tomé de Burguillos (1634) ${ }^{1}$
}

\author{
Antonio Sánchez Jiménez \\ Université de Neuchâtel \\ Antonio.sanchez@unine.ch
}

Recepción: 13/11/2020, Aceptación: 21/05/2021, Publicación: 22/12/2021

\begin{abstract}
Resumen
El presente estudio examina dos complejos pasajes de las Rimas de Tomé de Burguillos, ejemplos respectivos, pero elusivos, de polémica y burla, o más bien de burla con polémica y sin ella. En el primero, incluido en La Gatomaquia, estudiamos el sentido de una maliciosa referencia al poeta Anastasio Pantaleón de Ribera, ya difunto en 1634. En el segundo, el soneto 127 (129 en la edición de Carreńo), mostramos el blanco satírico de otra referencia sarcástica, alusión que le confiere al poema un tono burlesco acorde con el de muchos otros sonetos de la colección. Para llevar a cabo nuestro estudio nos ceñimos a una metodología de comentario de texto. Tras examinar las lecturas de la crítica, y en especial las de los editores de los versos en cuestión, presentamos la nuestra, que ilustra el conceptismo de los pasajes poniéndolos en contraste con diversos lugares de obras de Lope y de otros escritores del momento.
\end{abstract}

Palabras clave

Lope de Vega; Rimas de Tomé de Burguillos; sátira; polémica; burla.

\footnotetext{
Abstract

English Title. Lope de Vega, Tobacco, and Cuckoldry: Two Notes on Polemics and Mock Poetry in the Rimas de Tomé de Burguillos (1634).
}

1. Este texto se inscribe en el proyecto "Lope de Vega as a Courtly Writer: La Filomena (1621) and La Circe (1624)" (IZSAZ1_173356 / 1), financiado por el Fonds National de la Recherche Scientifique (FNS) de la Confederación Helvética. 
The present article examines two complex passages in Lope de Vega's Rimas de Tomé de Burguillos, respective and elusive examples of polemics and mock poetry, or, rather, of polemical and non-polemical mock poetry. In the first, included in La Gatomaquia, we study the meaning of a malicious reference to the poet Anastasio Pantaleón de Ribera, already dead in 1634. In the second, sonnet 127 (129 in Carreño's edition), we show the satirical target of another sarcastic reference, an allusion that gives the poem its burlesque tone, parallel to that of many texts in the book. In order to achieve our objective, we use a textual commentary methodology. After reviewing the critics' opinions on the passages in question, and in particular their editors', we present ours, which illustrate the passages conceptismo by contrasting them with several loci in Lope's works and in other writers of the time.

Keywords

Lope de Vega; Rimas de Tomé de Burguillos; satyre; polemics; mock poetry.

\section{Introducción: polémica y sátira}

Las polémicas poéticas proliferaron en el Siglo de Oro, enfrentando, según la época, a grandes huestes ordenadas (cultos contra llanos, por ejemplo), a campeones individuales o a todos contra todos, como en una folla caballeresca. En cualquier caso, los poetas necesitaban participar en ellas para perfilarse en el campo literario: la polémica mostraba el ingenio en el ataque y la defensa, pero también establecía fronteras y definía grupos, separando enemigos de aliados. Desde muy pronto, Lope de Vega albergó la ambición de dominar ese belicoso campo y de monopolizar los laureles de Apolo, por lo que se vio implicado en numerosas polémicas. Nos referimos a las que estudió Entrambasaguas (1967: I, 63-580; II, 11-411) y han cartografiado trabajos más recientes, ${ }^{2}$ que examinan

2. Recordemos el congreso dedicado al Lope polemista (Prolope, 2008) y los volúmenes del Anuario Lope de Vega resultado del mismo (2008 y 2009). 
las disputas de Lope contra unos ingenios sevillanos (Rico García y Solís de los Santos 2008), contra Diego de Colmenares (Tubau 2007; 2008), contra el autor de la Spongia (González-Barrera 2011; Conde Parrado y Tubau 2015), contra Jáuregui (Romanos 1978: 38-39; Montero 2008: 202) o contra el cronista real y erudito cultista José de Pellicer (Rozas 1990: 133-168; Iglesias Feijoo 2001; Sánchez Laílla 2008; Núnez Rivera 2019), por no hablar de las decisivas intervenciones del Fénix en la polémica por excelencia de nuestro Siglo de Oro, la gongorina. El papel central de la polémica en las letras áureas explica que sus matices tiñan incluso el último libro que Lope publicó en vida, las Rimas humanas y divinas del licenciado Tomé de Burguillos (1634), que estudiosos como Rozas (1990) han entendido, tal vez forzando el texto, como una especie de gran crítica contra Pellicer, esto es, como un gran texto polémico.

Obviamente, muchos de los poemas polémicos del Fénix tenían carácter paródico, satírico y burlesco, modalidades que también resultaban imprescindibles para hacerse un lugar en el campo de las letras del momento. Es más, concretamente la sátira, aunque censurara defectos morales, más que literarios, resulta esencial para entender las disputas literarias del Siglo de Oro, pues permitía a los poetas forjarse un perfil moralizante digno y acorde con sus intereses. $\mathrm{O}$ al menos con los intereses cortesanos de Lope, quien llevaba desde comienzos del reinado de Felipe IV tratando de presentarse como un escritor moral y moralizante y quien, por tanto, recurrió frecuentemente a la sátira en sus ańos finales. ${ }^{3}$ Asimismo, la burla con nula o muy somera pretensión moral también está presente en el Burguillos, donde la ampara la premisa satírica y polémica del libro: recordemos que los poemas los enuncia Tomé de Burguillos, un personaje desengańado y cansado de ver que los laureles y mecenazgo que él merece se los lleven otros poetastros, cultistas o no. Por tanto, sátira (moral y literaria) y burla son dos de los polos principales del libro de 1634.

El presente estudio examina dos complejos pasajes de las Rimas de Tomé de Burguillos, ejemplos respectivos, pero elusivos, de polémica y burla, o más bien de burla con polémica y sin ella. En el primero, incluido en La Gatomaquia, estudiaremos el sentido de una maliciosa referencia al poeta Anastasio Pantaleón de Ribera, ya difunto en 1634. En el segundo, el soneto 127 (129 en la edición de Carreńo), mostraremos el verdadero blanco satírico de otra referencia sarcástica, alusión que le confiere al poema un tono burlesco acorde con el de muchos otros sonetos de la colección. Para llevar a cabo nuestro estudio nos ceñiremos a una metodología de comentario de texto, estudiando en detalle la construcción retórica de los poemas. Tras examinar las lecturas de la crítica, y en especial las de los editores de los versos en cuestión, presentaremos la nuestra, que ilustrará el conceptismo de los pasajes poniéndolos en contraste con diversos lugares de obras de Lope y de otros escritores del momento.

3. Para los intentos cortesanos de los años veinte son esenciales La Filomena (1621) y La Circe (1624). 


\section{Sífilis y tabaco}

Como hemos adelantado, el primer lugar que vamos a analizar se encuentra en La Gatomaquia, zoomaquia ${ }^{4}$ sobre las disputas bélicas y amorosas de unos felinos en los tejados de Madrid. Lope la incluyó en las Rimas de Tomé de Burguillos y es una de sus obras no dramáticas más y más justamente celebradas, hasta el punto que no hay lector que se resista a su encanto. Su fama alimenta la del Burguillos, que es, hoy por hoy, el libro del Fénix con más ediciones críticas: incluso si no contamos la de Blecua (1989), ${ }^{5}$ en lo poco que llevamos de siglo XXI se agolpan las de Carreño (2002), Rozas y Cañas Murillo (2005), Cuiñas Gómez (2008) y Arellano (2019), quien, además, ha dedicado una monografía al libro (Arellano 2012). Casi igual fortuna ha vivido La Gatomaquia, texto que no solamente se halla en todas las ediciones citadas (menos la de Rozas y Cañas Murillo), sino que también podemos encontrar en las de Gasparetti (1932), Rodríguez Marín (1935), del Campo (1948) y Sabor de Cortazar (1982), amén de, otra vez, Arellano (2020), en reimpresión de la parte correspondiente de la edición de 2019. ${ }^{6}$

En el texto que fija Sabor de Cortazar (1982), el pasaje que nos interesa es el siguiente:

Mas vuelve, oh, Musa, tú, para que pueda

ayudarme el favor de tu gimnasio,

que para lo que queda,

aunque parece poco,

al señor Anastasio

Pantaleón de la Parrilla invoco,

porque de su tabaco

me dé siquiera cuanto cubra un taco. (VII. 145-152)

El imperativo inicial ("vuelve") nos revela que los versos en cuestión aparecen en el áphodos o fórmula de retorno de una digresión (sed redeo ad propositum) (Sobejano 1983: 469). En ella, el poeta ha discurrido por todo lo divino y lo humano, incitado por la mención del minúsculo caballo de uno de los gatos

4. Tomamos el término de Bonilla Cerezo y Luján Atienza (2014), quienes también pasan revista a otros marbetes empleados por la crítica previa, tal como "épopées animalières" (Fasquel 2010: 589) o "zooépica" (Cacho Casal 2013). A su vez, esta segunda propuesta ("zooépica”) bebe de dos modelos previos: por una parte, el "komische Tierepos" de Schmidt (1953: 45-61), un clásico en el estudio de la epopeya burlesca; por otra, la "zooepica” de Zaggia (2013).

5. Esta edición del Burguillos no es exenta (está en unas Obras poéticas selectas) y solo presenta una anotación esporádica, pero es un trabajo muy valioso porque propone enmiendas que, en su mayoría, han adoptado los críticos posteriores.

6. Además, La Gatomaquia es el objeto de una monografía del padre Blázquez Rodrigo (1995) — por desgracia rea de diversos plagios (Luján Atienza 2002: 11) - y una larga serie de artículos que no vamos a reseñar. 
(VII. 66). Esta maravilla le ha llevado a hablar de las alas de Pegaso, de los pigmeos y trogloditas de Tracia, de la naturaleza de los pigmeos y sus batallas contra las grullas (con menciones de Plinio, Homero, Estacio, Mela, Aristóteles, san Agustín y Juvenal), de la pintura en miniatura o de la licencia para mentir de los poetas (con el tópico ejemplo del libro IV de la Eneida). Tras toda esta cómica balumba, el distraído poeta le pide a su musa que le ayude a regresar a la materia en cuestión (la guerra gatuna), pidiendo tanto su inspiración como, y es lo que nos interesa, el furor poético que le pueda proporcionar una mínima cantidad del tabaco de "Anastasio / Pantaleón de la Parrilla".

Aunque con comprensible cautela, los críticos han aventurado que tras este misterioso nombre se esconde una cómica alusión al divertido poeta Anastasio Pantaleón de Ribera (Gasparetti 1932: 139-140; Rodríguez Marín 1935: 237; del Campo 1948: 234; Blecua 1989: 1406; Sabor de Cortazar 1982: 219; Carreño 2002: 489; Cuiñas Gómez 2002: 550-551; Arellano 2019: 671-672; 2020: 183). Además, los estudiosos recuerdan que, aunque Anastasio Pantaleón había muerto en 1629, sus obras acababan de imprimirse precisamente en 1634, y nada menos que al cuidado de Pellicer (Rodríguez Marín 1935: 237). Por ello, habrían llamado la atención del Fénix, quien además las pudo consultar tranquilamente porque el volumen tiene fe de erratas y tasa de abril de 1631, aunque solo apareciera impreso en 1634. De hecho, La Barrera (1890: 474) sugiere que el "no sacadas de biblioteca ninguna (que en castellano se llama librería)" del subtítulo del Burguillos es una alusión burlesca al libro de Anastasio Pantaleón y Pellicer, que se llamaba "Obras de Anastasio Pantaleón. Salen a la luz de la biblioteca de don José Pellicer" (f. 1r). ${ }^{7}$

Obviamente, la relación entre el Fénix y Pellicer justifica ya la posibilidad de una alusión burlesca, aunque fuera a costa de un escritor muerto con quien, además, Lope no se llevaba bien (Brown, 1981). Anastasio Pantaleón era poeta gongorino y amigo de Pellicer (Rozas 1990: 159; Ponce Cárdenas 2001: 87-96; 2010: 25), y Lope había aludido maliciosamente a él en el Laurel de Apolo (silva VII, vv. 503-508) (Ponce Cárdenas 2007: 123), como han subrayado los críticos: Entrambasaguas (1967: I, 243) considera esa mención "enrevesada y [de] cruel intención en el fondo"; Carreño (2002: 489; 2007: 382), "irónica”, y Cuiñas Gómez (2008: 551), "fría y algo envenenada". Además, los citados Entrambasaguas y Cuiñas Gómez suponen que la mención del Laurel de Apolo fue la respuesta de Lope a una alusión burlesca de Pantaleón al Fénix en el poema que se titula "Pidiendo al excelentísimo señor conde de Saldaña un corte de un vestido de paño que le ofreció al poeta, y enviándole un vidro de camuesas en conserva”. Fuera por esos versos o por las razones arriba aludidas (el gongorismo de Pantaleón, su amistad con Pellicer), lo cierto es que Lope tenía ojeriza a Pantaleón.

7. Burguillos vuelve a reírse de la palabra en el soneto 116: "porque es llamar al guante quiroteca, / esto de biblioteca o bibliotaca" (vv. 7-8). 
En La Gatomaquia, esta inquina se manifiesta en una broma relacionada con la terrible y vergonzosa enfermedad de que adolecía Pantaleón: la sífilis. Probablemente, la cura a la que se sometió el poeta hacía necesario el uso del cauterio, lo que convertía al escritor en un potencial chorizo a la parrilla. Ponce Cárdenas (2007: 124) aclara así el chiste invocando una referencia intertextual:

La alteración del nombre, que figura como "Anastasio Pantaleón de la Parrilla", se inspira en un romance del poeta fallecido, donde este se refiere así a su propia sífilis: "Culpa tiene el cocinero, / que, creyéndome chorizo, / me espetó, de mis humores / alterando el equilibrio" (Ms. 3941, f. 84r). El chiste culinario de Lope ("Pantaleón de la Parrilla") se explica, por tanto, con el peculiar martirio que sufriera el joven [Pantaleón de la Ribera] durante su enfermedad. Sobre la críptica referencia al tabaco, no tenemos información alguna que afirme que Pantaleón había adquirido el hábito de fumar.

Antes, Brown (1981: 693) entendía también que el pasaje hacía relación a las curas de la sífilis de Pantaleón, aunque se muestra menos prudente que Ponce Cárdenas y toma la mención del tabaco como un reflejo de las costumbres del poeta: "Lo que en efecto aprendemos de estos versos es que Anastasio tenía otro vicio: el de respirar polvo de tabaco, hábito muy común del siglo xvir".

Sin embargo, y he aquí nuestra aportación, lo cierto es que en los versos de Lope el tabaco no alude a los hábitos del poeta rival, sino a la terapia a la que Pantaleón tenía que someterse. Durante el Siglo de Oro esa planta de origen americano se usaba para paliar los dolores de la sífilis, según señalaba Fernández de Oviedo en su Historia general y natural de las Indias: "Sé que algunos cristianos ya lo usan, en especial algunos que están tocados del mal de las bubas, porque dicen los tales que en el tiempo que están así transportados no sienten los dolores de su enfermedad" (Ortiz 1978: 222). Esta propiedad y práctica curativa estaba muy extendida y la confirman otros tratados de la época (Castro, Historia, f. 72r), lo que explica su aparición en el misterioso pasaje de La Gatomaquia. En él, Lope sugiere malévolamente que para escribir Anastasio Pantaleón se inspiraba inspirando tabaco (literalmente, y valga el juego de palabras). Además, en el Burguillos el Fénix asocia esa planta con los desatinos de los cultos (núm. 122, v. 10), ${ }^{8}$ pues, en efecto, en la época se creía que el tabaco provocaba diabólicas visiones (Monardes, Segunda, ff. 19r-19v). Por tanto, los versos en cuestión son, en efecto, arteros, y fustigan tanto al alegre Pantaleón como a los cultos, aunque con mayor malicia de la que se creía hasta ahora.

8. Citamos el Burguillos por la edición de Carreño (2002). La numeración en las otras ediciones modernas es distinta: para encontrar en ellas el poema en cuestión basta con restarle dos al número de Carreño. 


\section{Carneros y cuernos}

En cuanto al segundo pasaje que nos interesa, se encuentra en el soneto 129 del Burguillos, que copiamos a continuación alterando levemente la puntuación de Carreño:

$A$ un amigo del poeta que iba fuera de buena gana

Galán de verde vas, hermano Alcino; pájaro mudas: buenas dichas hayas; pues con lo verderón te apapagayas, ¡notable comisión, bravo camino!

Bien te parece el traje montesino para entre cabrahígos y altas hayas; vuelvas más alto, aunque también lo vayas, que Lanzarote de Bretaña vino.

Como un Orlando vas determinado; lo verde es esperanza: no se pierde, y más en los que viven sin cuidado.

Pero dice que vas quien siempre muerde más que para galán, para guisado, porque pudieras ser carnero verde.

Al respecto, Blecua (1989: 1309) solo explica que el "carnero verde" es un guiso de carnero con ajo y perejil, lo que también documenta Carreño (2002: 314), quien añade que el verde es el color de la esperanza (v. 10). Por su parte, Rozas y Cañas Murillo (2005: 292-293) solo aportan documentación sobre las diversas referencias al romancero que hay en el soneto (vv. 5 y 8), ${ }^{9}$ amén de una hipótesis sobre el adjetivo "determinado": en su opinión, sería un recuerdo de "la continuación del texto de Boyardo hecha por Martín de Bolea y Castro, que se titulaba Orlando determinado". Cuiñas Gómez (2008: 362-363) añade que los trajes verdes se solían usar para ir de camino, detalle en el que abunda Arellano (2019: 473). En sus escolios sobre el texto, el propio Arellano (2012: 200-201) glosa las ediciones previas (C: Carreño; RC: Rozas y Cañas Murillo; CG: Cuiñas Gómez):

En el 127 se dirige a un amigo que se ha vestido de verde para hacer un viaje. Las notas de C y RC bastarán para comprender algunas alusiones, no así las de CG, que

9. Sin embargo, aunque Montesinos es personaje de diversos romances carolingios, en el que señalan Rozas y Cañas Murillo (Durán, Romancero general, núm. 370, pp. 237-240) no hay nada en común con el soneto lopesco. Por ello, Arellano (2019: 473) indica, creemos que con razón, que el adjetivo "montesino" quiere decir aquí 'montaraz' y no alude al personaje. 
anota defectuosamente el chiste con "carnero verde", término de comparación cómico para el galán vestido de ese color (carnero verde es un tipo de guisado; chiste culinario habitual en la literatura jocosa). Es mala puntuación la de C y CG en el v. 1: "Galán, de verde vas, hermano Alcino", y buena la de RC: "Galán de verde vas, hermano Alcino", donde "galán” no es sustantivo en forma vocativa, sino adjetivo ('vas elegante y bizarro vestido de verde').

El comentario sobre la puntuación es acertado, pero Arellano no acaba de explicar el soneto, que él y todos los editores parecen entender (ninguno lo hace explícito) como una burla del traje de camino del personaje, a quien se compararía con un verderón, con un papagayo y, finalmente, con un guiso con perejil, el mentado "carnero verde".

El chiste es demasiado inocente (y frío) para sustentar un soneto en este libro, lo que ya hace sospechar que hay ironía o malicia en algunos pasajes, aunque, obviamente, el lector necesita algunas claves para detectarla. Estas claves no se encuentran, desde luego, en el epígrafe, al menos en una primera lectura. La ficción del libro es que estos titulillos los escribe Lope, en su función de editor de los poemas de su amigo Burguillos, y muchos de esos epígrafes son reveladores. Sin embargo, el presente solo indica quién es el Alcino a quien se dedica el poema ("un amigo del poeta"), aclara que el personaje "iba fuera" y que cuando viajaba lo hacía "de buena gana". Repetimos que la lectura maliciosa de esta explicación del "editor” se encontrará según avance el poema.

De modo semejante, el primer cuarteto parece inocente, aunque encontramos en él una mención que podría hacer sospechar a algunos lectores. El primer verso describe la escena en el contexto de un elogio al aspecto de Alcino, nombre genérico, propio para un poema burlesco o satírico. Alcino se ha vestido de verde para ir de viaje y el poeta encomia su elegancia. Luego, el segundo aprovecha la imagen del cambiar de traje para centrarse en el mundo de las aves, las cuales mudan su plumaje como hace Alcino, quien pasa de uno (el negro de cortesano, que evocaría un pájaro negro) a otro (el verde de viaje, propio de otras aves), en lo que el poeta le desea suerte ("buenas dichas hayas"). El tercer verso menciona a dos de estas aves, las dos parleras (Plinio, Historia, p. 795) y, sobre todo, verdes, en referencia al color del traje de Alcino: el verderón y el papagayo. ${ }^{10} \mathrm{El}$ neologismo "apapagayas" remite, obviamente, al papagayo, cuya apariencia imita Alcino al vestirse de verde. ${ }^{11}$ Además, este pájaro nos delata en qué pensaba

10. Que en las Indias los había verdes cuenta Huerta en su traducción de Plinio (Historia, p. 799). 11. Además de estos personajes lopescos que vamos a desglosar, hay otros cervantinos que también se asocian al papagayo, aunque no se relacionan con el tema que nos interesa. Uno es Vidriera en "El licenciado Vidriera", pues el narrador cuenta que "Habíase vestido Tomás de papagayo, renunciando los hábitos de estudiante” (Novelas ejemplares, p. 270). En este caso, la referencia no es al color verde, sino a la abigarrada vestimenta de los soldados, que es la que aquí adopta Vidriera. Otro es don Diego de Miranda, este sí de verde (“Caballero del Verde Gabán”). En unos pasajes célebres, Márquez Villanueva (1975: 227) explica que se viste de "pagapayo" (la metáfora es de Márquez 
Lope a estas alturas del poema, pues entre las referencias a papagayos de su amplia obra encontramos dos bastante reveladoras para entender nuestro texto. La primera aparece en Los comendadores de Córdoba, en el terrible castigo final del marido engañado:

Desmayose mi mujer;

déjela para más pena,

y discurriendo la casa,

maté cuantos hubo en ella:

a don Fernando, a dońa Ana,

dos dueñas, cuatro doncellas,

pajes, escuderos, mozas,

lacayos, negros y negras,

los perros, gatos y monas,

hasta un papagayo, que era

también traidor, pues hablaba

y no me dijo la afrenta. (vv. 2970-2981)

Esta asociación entre el papagayo con el callar ante un caso de cuernos también aparece, años más tarde, en El cuerdo en su casa (c. 1606-1608) (Morley y Bruerton 1968: 307), en referencia intertextual a Los comendadores de Córdoba:

Que de un cierto Veinticuatro

hay una historia espantosa,

de corónicas en prosa

y versos en el teatro:

este dicen que mató

las criadas y criados,

o inocentes o culpados;

tanto, que no perdonó

a un papagayo que hablaba,

porque no se lo decía. ${ }^{12}$ (vv. 2902-2911)

Abajo volveremos a esta temática, pues ahora falta por revisar el cuarto verso, una nueva exclamación de encomio y sorpresa por el viaje de Alcino.

El segundo cuarteto prosigue alabando la apariencia del personaje con el traje de camino ("Bien te parece el traje montesino"), aunque puntualiza que este es bueno para el campo e introduce para describirlo una planta sospechosa:

Villanueva, no de Cervantes) y le asocia a la locura, pues, según sostiene, los bufones podían vestir de verde. Esta conexión del verde con la locura podría ser sugerente para el pasaje que nos interesa, pues, como veremos, este Alcino da ciertas muestras de inconsciencia (no de locura).

12. Hay un episodio parecido en una comedia de Lope de 1600: La contienda de García de Paredes y el capitán Juan de Urbina, pero en él Juan de Urbina mata a su mujer (adúltera), criados, gatos y perros (vv. 1786-1794 y 2624-2635), sin mención de papagayos. 
los cabrahígos. Observemos al respecto otra aparición de este árbol en el Burguillos, y concretamente en La Gatomaquia, en una digresión sobre Angélica y Medoro, quienes escriben

en el papel de las cortezas tiernas de aquellos olmos, de su bien testigos, para el francés Orlando cabrahígos. (silva IV, vv. 339-341)

Como explica Rodríguez Marín (1935: 170) en su edición de La Gatomaquia, el cabrahígo es una planta como otra cualquiera, pero su nombre contiene la palabra cabra y es, por tanto, propia para alusiones a amantes burlados, como el Orlando de estos versos. Los indicios funestos se acumulan, pues, para Alcino, pues si el mentado papagayo se posa sobre un cabrahígo parecerá más un cuco que otra cosa. Es, en todo caso, lo que sugiere el maliciosísimo verso 7: el adjetivo que Burguillos había aplicado en el verso 6 a las hayas ("altas") se adjudica ahora, en forma de deseo, a Alcino. En este contexto fomentado por los cabrahígos, el verso no expresa una esperanza inocente, pues lo que hará crecer a Alcino en su viaje ("vuelvas más alto") serán los cuernos, que medrarán en su ausencia, aunque el personaje ya esté bien servido al partir ("aunque también lo vayas"). En cambio, el eco del romance de Lanzarote en el verso 8 no nos parece alusivo a los cuernos y otorga un punto absurdo al soneto, aunque no cabe descartar que la materia de Francia (tal vez "montesino", seguro "Orlando") atraigan el recuerdo de Lanzarote, Ginebra y Arturo, por más que en este trío no fuera precisamente Lanzarote el engańado.

Los tercetos aclaran esta burla con la alusión a Orlando, pues la figura del paladín francés sugiere que también la resolución de ausentarse de Alcino acabará en cuernos. Además, la conexión entre Orlando y los cabrahígos, arriba indicada y evidente en La Gatomaquia, ilumina el sentido del pasaje. Desde ese ángulo, la verde esperanza de Alcino y su vivir "sin cuidado" ('sin preocupación') resulta ingenua, suicida casi.

Lo que hasta el momento han sido equívocos más o menos evidentes se convierten en certezas en el último terceto, donde Burguillos introduce una nueva voz, la de un cínico murmurador ("quien siempre muerde") que enuncia en estilo indirecto el concepto final: la verdura y los cuernos de Alcino le hacen parecer más un guiso de carnero verde que un galán. En este punto, la acumulación de indicios en crescendo de claridad (papagayos, cabrahígos, altura, Orlando) provocarían la carcajada final, con la aparición del meridiano apelativo de "carnero". A su luz, la despreocupación de Alcino al irse fuera "de buena gana" resulta imprudente, pues da ocasión a su amante o cónyuge de ponerle los cuernos que ya ostenta el ufano personaje.

En suma, el soneto 129 es burlesco y trata de los cuernos, tema, por cierto, presente en el Burguillos como en casi toda la poesía burlesca del momento. Los encontramos en el soneto 41 del Burguillos ("¡Mal haya el hombre que de cuer- 
nos fía!", v. 14) y también en textos tempranos como el soneto CXV de las Rimas (Sánchez Jiménez 2014), en este enlazados en una red de referencias astrológicas que aclara un "Capricornio" final que cumple la función de nuestro "carnero" del Burguillos.

\section{Conclusión}

En trabajos recientes, Arellano (2012 y 2019) ha insistido en la dificultad de las Rimas de Tomé de Burguillos, obra que, aunque no es cultista, ostenta un conceptismo que exige cierto esfuerzo intelectual por parte del lector. En este trabajo, lo hemos mostrado con dos notas a sendos pasajes del libro, lugares de sesgo diverso (uno polémico, burlesco el otro), pero que tienen en común un conceptismo que esconde referencias escatológicas, en el primer caso para denigrar a Anastasio Pantaleón y los poetas cultos, en el segundo para preparar una broma ingeniosa. El mecanismo de alusiones es también semejante, así como el método que hemos empleado para dilucidarlas, que ha sido contrastar los pasajes con otros de Lope (en el propio Burguillos, en algunas de sus comedias) en un comentario de texto. Asimismo, hemos señalado que estos tonos están relacionados porque en el Burguillos Lope enuncia el libro a través de un heterónimo burlesco que le otorga gran libertad y que le permite asestar golpes escatológicos a rivales como Pellicer, Anastasio Pantaleón o los cultos en general. Sin embargo, los dos pasajes que hemos analizado también nos recuerdan la característica central que suele separar los textos polémicos (normalmente también burlescos) de los simplemente jocosos: mientras estos últimos se pueden descifrar recurriendo a códigos culturales extendidos en el momento (la burla genérica de los cornudos, el doble sentido de ciertas palabras), los primeros suelen exigir un conocimiento preciso de circunstancias concretas de la corte y de las relaciones entre los poetas. En efecto, el lector que no supiera que Anastasio Pantaleón era sifilítico jamás entendería la broma de Lope. En ese sentido, si el conceptismo en general sirve para distinguir entre lectores agudos y romos, el conceptismo mezclado con las referencias particulares del mundillo de los poetas separa a los lectores informados de los que no lo están, en un fenómeno que es parte de la formación del campo literario del momento. 


\section{Bibliografía}

Anuario Lope de Vega, XIV (2008).

-, XIV (2009).

Arellano, Ignacio, El ingenio de Lope de Vega. Escolios a las Rimas humanas y divinas del licenciado Tomé de Burguillos, Nueva York, IDEA, 2012.

- (ed.), Lope de Vega Carpio, Rimas humanas y divinas del licenciado Tomé de Burguillos, Madrid, Iberoamericana, 2019.

- (ed.), Lope de Vega Carpio, La Gatomaquia, en Antología de la literatura burlesca del Siglo de Oro. Volumen 1. Poesía de Lope de Vega, Góngora y Quevedo, Nueva York, IDEA, 2020, pp. 93-192.

Blázquez Rodrigo, Marcelo, La Gatomaquia de Lope de Vega, Madrid, CSIC, 1995.

Blecua, José Manuel (ed.), Lope de Vega Carpio, Rimas humanas y divinas del licenciado Tomé de Burguillos, en Obras poéticas, Barcelona, Planeta, 1989, pp. 1127-1447.

Bonilla Cerezo, Rafael, y Ángel L. Luján Atienza (eds.), Zoomaquias. Épica burlesca del siglo XVIII, Madrid, Iberoamericana, 2014.

Brown, Kenneth, "Lope frente a Anastasio Pantaleón", en Lope de Vega y los orígenes del teatro español: actas del I Congreso Internacional sobre Lope de Vega, ed. Manuel Criado de Val, Madrid, Edi-6, 1981, pp. 687-694.

Cacho Casal, Rodrigo, "Una variante eroicomica: la tradizione zooepica in Spagna e la Muracinda di Juan de la Cueva", en L'eroicomico dall'Italia all'Europa. Atti del convegno Università di Losanna, 9-10 settembre 2011, ed. Gabriele Bucchi, Pisa, ETS, 2013, pp. 137-154.

Campo, Agustín del (ed.), Lope de Vega Carpio, La Gatomaquia, Madrid, Castilla, 1948.

Carreño, Antonio (ed.), Lope de Vega Carpio, Rimas humanas y divinas del licenciado Tomé de Burguillos, Salamanca, Almar, 2002.

- (ed.), Lope de Vega Carpio, Laurel de Apolo, Madrid, Cátedra, 2007.

Castro, Juan de, Historia de las virtudes y propiedades del tabaco, Córdoba, Salvador de Cea Tesa, 1620.

Cervantes, Miguel de, Novelas ejemplares, ed. Jorge García López, Madrid, RAE, 2013.

Conde Parrado, Pedro, y Xavier Tubau Moreu (eds.), Expostulatio Spongiae. En defensa de Lope de Vega, Madrid, Gredos, 2015.

Cuiñas Gómez, Macarena (ed.), Lope de Vega Carpio, Rimas humanas y divinas del licenciado Tomé de Burguillos, Madrid, Cátedra, 2008.

Durán, Agustín, Romancero general, I, Madrid, Rivadeneyra, 1877.

Entrambasaguas, Joaquín de, Estudios sobre Lope de Vega, Madrid, Consejo Superior de Investigaciones Científicas, 1967, 3 vols.

Fasquel, Samuel, "Le barde et le bouffon. La geste burlesque à l'époque de Lope de Vega", Bulletin Hispanique, CXII (2010), pp. 587-632. 
Gasparetti, Antonio (ed.), Lope de Vega Carpio, La Gatomaquia, Florencia, La Nuova Italia, 1932.

González-Barrera, Julián, Expostulatio Spongiae. Fuego cruzado en el nombre de Lope, Kassel, Reichenberger, 2011.

Iglesias Feijoo, Luis, "Sobre la fecha de una comedia de Lope y su guerra con Pellicer", en Prosa y poesía: homenaje a Gonzalo Sobejano, ed. Christopher Maurer, Jean-François Botrel, Yvan Lissorgues y Leonardo Romero Tobar, Madrid, Gredos, 2001, pp. 171-188.

La Barrera, Cayetano Alberto de, Nueva biografía de Lope de Vega, Madrid, Real Academia Española, 1890.

Luján Atienza, Ángel Luis (ed.), José de Villaviciosa, La Moschea. Poética inventiva en octava rima, Cuenca, Diputación de Cuenca, 2002.

Márquez Villanueva, Francisco, Personajes y temas del Quijote, Madrid, Taurus, 1975.

Monardes, doctor, Segunda parte del libro de las cosas que se traen de nuestras Indias Occidentales que sirven al uso de medicina, Sevilla, Alonso Escribano, 1571.

Montero, Juan, "La rivalidad literaria entre Lope y Jáuregui", Anuario Lope de Vega, XIV (2008), pp. 181-212.

Morley, S. Griswold, y Courtney Bruerton, Cronología de las comedias de Lope de Vega. Con un examen de las atribuciones dudosas, basado todo ello en un estudio de su versificación estrófica, trad. María Rosa Cartes, Madrid, Gredos, 1968.

NúNEEz Rivera, Valentín, "Lope, editor de poesía: la dispositio polémica de las Rimas de Tomé de Burguillos", en Controversias y poesia: (de Garcilaso a Góngora), ed. Mercedes Blanco y Juan Montero Delgado, Sevilla, Universidad de Sevilla, 2019, pp. 287-316.

Ortiz, Fernando, Contrapunteo cubano del tabaco y el azúcar, Caracas, Ayacucho, 1978.

Pantaleón de Ribera, Anastasio, Obras de Anastasio Pantaleón de Ribera, Madrid, Francisco Martínez, 1634.

Puinio Segundo, Cayo, Historia natural de Cayo Plinio Segundo, traducida por el licenciado Jerónimo de Huerta, Madrid, Luis Sánchez, 1624.

Ponce Cárdenas, Jesús, Góngora y la poesía culta del siglo XVII, Madrid, Laberinto, 2001.

—, "De burlas y enfermedades barrocas: la sífilis en la obra poética de Anastasio Pantaleón de Ribera y Miguel Colodrero de Villalobos”, Criticón, núm. 100 (2007), pp. 115-142.

—, "Pantaleón de Ribera, Anastasio", en Diccionario filológico de literatura española (siglo XVII), ed. Pablo Jauralde Pou, Delia Gavela y Pedro C. Rojo Alique, Madrid, Castalia, 2010, 2 vols., II, pp. 25-31.

Rico García, José Manuel, y José Solís de los Santos, "La sonetada a Lope del Cartapacio de Palomo", Anuario Lope de Vega, XIV (2008), pp. 235-268. 
Rodríguez Marín, Francisco (ed.), Lope de Vega Carpio, La Gatomaquia, Madrid, C. Bermejo, 1935.

Romanos, Melchora (ed.), Juan de Jáuregui, Discurso poético, Madrid, Editora Nacional, 1978.

Rozas, Juan Manuel, Estudios sobre Lope de Vega, Madrid, Cátedra, 1990.

Rozas, Juan Manuel, y Jesús Cañas Murillo (eds.), Lope de Vega Carpio, Rimas humanas y divinas del licenciado Tomé de Burguillos, Madrid, Castalia, 2005.

Sabor de Cortazar, Celina (ed.), Lope de Vega Carpio, La Gatomaquia, Madrid, Castalia, 1982.

SÁnchez Jiménez, Antonio, "Algunos chistes astrológicos de Lope de Vega", Criticón, núm. 122 (2014), pp. 41-52.

SÁnchez Laílla, Luis, “'Oh estudio liberal, discreto amigo’: Lope y la apología del sabio", Anuario Lope de Vega, XIV (2008), pp. 291-342.

Schmidt, Karlenst, Vorstudien zu einer Geschichte des komischen Epos, Halle, Niemeyer, 1953.

Sobejano, Gonzalo, "La digresión en la prosa narrativa de Lope y en su poesía epistolar", en Estudios ofrecidos a Emilio Alarcos Llorach, ed. Emilio Alarcos Llorach y M. V. Conde, Oviedo, Universidad de Oviedo, 1983, II, pp. 469-494.

Tubau, Xavier, Una polémica literaria. Lope de Vega y Diego de Colmenares, Madrid, Iberoamericana, 2007.

-, Lope de Vega y las polémicas literarias de su época: Pedro de Torres Rámila y Diego de Colmenares, Tesis doctoral, Bellaterra, Universitat Autònoma de Barcelona, 2008.

Vega Carpio, Lope de, Los comendadores de Córdoba, ed. José Enrique Laplana Gil, en Comedias de Lope de Vega. Parte II, coord. Silvia Iriso, Lérida, Milenio, 1998, 3 vols., II, pp. 1023-1174.

—, La contienda de García de Paredes y el capitán Juan de Urbina, en El Sansón de Extremadura: Diego García de Paredes en la literatura española del siglo XVI, ed. Antonio Sánchez Jiménez, Newark, Juan de la Cuesta, 2006, pp. 168345.

-, El cuerdo en su casa, ed. Laura Fernández y Rafael Ramos, en Comedias de Lope de Vega. Parte VI, coord. Victoria Pineda y Gonzalo Pontón, Lérida, Milenio, 2005, 3 vols., II, pp. 775-902.

—, La Gatomaquia, ed. Celina Sabor de Cortazar, Madrid, Castalia, 1982.

—, Laurel de Apolo, ed. Antonio Carreño, Madrid, Cátedra, 2007.

-, Rimas humanas y divinas del licenciado Tomé de Burguillos, ed. Antonio Carreño, Salamanca, Almar, 2002.

ZagGia, Massimo, "Per una storia del genere zooepico fra Quattro e Cinquecento: testi e linee di sviluppo", en L'eroicomico dall'Italia all'Europa. Atti del convegno Università di Losanna, 9-10 settembre 2011, ed. de Gabriele Bucchi, Pisa, ETS, 2013, pp. 27-53. 